
$\angle$ Research Square
Preprints are preliminary reports that have not undergone peer review.
They should not be considered conclusive, used to inform clinical practice, or referenced by the media as validated information.

\title{
The effect of duration time on the efficacy and safety during endoscopic papillary balloon dilation for choledocholithiasis treatment
}

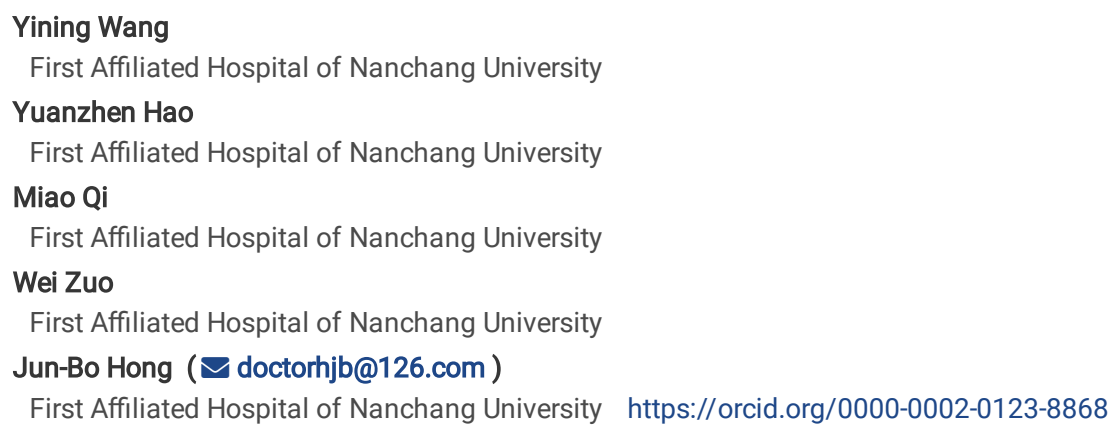

First Affiliated Hospital of Nanchang University

Yuanzhen Hao

First Affiliated Hospital of Nanchang University

Miao Qi

First Affiliated Hospital of Nanchang University

Wei Zuo

First Affiliated Hospital of Nanchang University

Jun-Bo Hong ( $\nabla$ doctorhjb@126.com )

First Affiliated Hospital of Nanchang University https://orcid.org/0000-0002-0123-8868

Research article

Keywords: EPBD, duration time, choledocholithiasis, post-ERCP complications

Posted Date: February 26th, 2020

DOI: https://doi.org/10.21203/rs.2.24607/v1

License: @) (7) This work is licensed under a Creative Commons Attribution 4.0 International License. Read Full License 


\section{Abstract}

Background

Endoscopic papillary balloon dilation (EPBD) has been widely accepted as an alternative to endoscopic sphincterotomy (EST) during therapeutic endoscopic retrograde cholangiopancreatography (ERCP) for choledocholithiasis treatment. No consensus has been established to define the precise duration of EPBD.

Methods

The PubMed, EMBASE, Cochrane Library database were systematically searched up to 31 May 2019 for research comparing short duration ( $\leq 30$ seconds) and long duration ( $\geq 1$ minute) of EPBD.

Results

Seven studies demonstrated that EPBD with long duration was superior than short duration in the stone clearance rate during the first ERCP (OR $0.61,95 \% \mathrm{CI}$ $[0.48,0.77], \mathrm{P}<0.001)$. Long duration exhibited a trend of higher efficacy in total stone removal across all ERCP sessions $(\mathrm{OR} 0.98,95 \% \mathrm{Cl}[0.73,1.31], \mathrm{P}=$ $0.89)$, less frequency of mechanical lithotripsy use (OR 1.22, 95\% $\mathrm{Cl}[0.79,1.89], \mathrm{P}=0.37)$. Compared with long duration, short balloon time had lower rate of overall complications $(\mathrm{OR} 0.85,95 \% \mathrm{Cl}[0.70,1.04], \mathrm{P}=0.11)$ and post-ERCP pancreatitis (OR $0.81,95 \% \mathrm{Cl}[0.63,1.03], \mathrm{P}=0.09)$. No significance was identified in hemorrhage $(\mathrm{OR} 0.86,95 \% \mathrm{Cl}[0.30,2.44], \mathrm{P}=0.77)$, perforation $(\mathrm{OR} 0.54,95 \% \mathrm{Cl}[0.14,2.14], \mathrm{P}=0.38)$ and cholangitis $(\mathrm{OR} 1.15,95 \% \mathrm{Cl}[0.80,1.66], \mathrm{P}=0.44)$.

Conclusion

Long duration ( $\geq 1$ minute) could significantly increase the efficacy of EPBD in stone removal rate during first ERCP session, manifesting higher rate of total CBD stone clearance and less mechanical lithotripsy. Short EPBD contributed to less overall complications than long EPBD but not significantly.

\section{Background}

Approximately $10 \%$ of the global adult are diagnosed with symptomatic gallstones and common bile duct stones (CBDS) accounted for $10 \%-15 \%$ symptomatic cholelithiasis[1]. For patients with choledocholithiasis, the therapeutic effect and prophylaxis has been obviously improved with the rapid progress of endoscopic techniques. Endoscopic sphincterotomy (EST) was firstly implemented in 1975 and gradually accepted as a common technique to enlarge the sphincter of Oddi (SO) during endoscopic retrograde cholangiopancreatography (ERCP)[2]. But EST also demonstrated failure of stone removal and post-procedure complications including post-ERCP pancreatitis (PEP), hemorrhage, perforation, cholangitis. As a potential method for preserving the function of SO, endoscopic papillary balloon dilation (EPBD) was invented and suggested as an alternative intervention to sphincterotomy for efficient and safe removal of CBDs with less hemorrhage events[3-7]. But higher incidence of PEP was reported in patients suffering EPBD. Recently, EPBD appears to be combined with preceding EST to treat large or difficult CBD stones, which could improve efficacy of stone removal and reduce the risk of related complications[8-11].

Although EPBD has been widely adopted for removal and extraction of CBD stones, no consensus of accurate duration time has been established. ERCP guideline of European Society of Gastrointestinal Endoscopy (ESGE) weakly recommends $\geq 2$-minute papillary dilation should be maintained after waist disappearance[4]. Endoscopic guideline for common bile duct stones of ESGE illustrated that EPBD duration should be maintained between 30 and 60 seconds from waist disappearance[12]. Conflicting conclusions were drawn from present published data. A network meta-analysis illustrated that EPBD with long dilation duration is safer than EPBD with short dilation duration and EST because $\leq 1$-minute dilation could increase the risk of PEP and long EPBD had a lower rate of overall complications[13]. Another meta-analysis indicated no significant difference in CBD stone clearance between endoscopic papillary large balloon dilation (EPLBD) and EST, despite EPLBD being taken with either short dilation time ( $\leq 30$ seconds) or long dilation time ( $\geq 1$ minute)[14]. Besides, it was reported that 30-second ballooning duration showed a lower tendency in the incidence of post-procedural pancreatitis than 2-minute ballooning duration[15].

Our meta-analysis and systematic review will aim to evaluate the efficacy and safety between short dilation duration ( $\leq 30$ seconds) or long dilation duration ( $\geq 1$ minute) during removal of $C B D$ stones by EPBD.

\section{Methods}

Our analysis was implemented on the basis of the Cochrane Handbook for Systematic Reviews of Interventions and presented according to Preferred Reporting Items for Systematic Reviews and Meta-analyses guidelines[16].

\section{Literature search}

We searched and identified research from PubMed, EMBASE, and Cochrane Library (including CENTRAL) database from the January $1 \mathrm{st}$, 1990 to May 31 st, 2019. Besides, we broaden the search for available or similar studies from review articles and academic conferences. No language restriction was achieved during our searching. The following keywords involved in our searching were "endoscopic retrograde cholangiopancreatography", "ERCP", "endoscopic papillary balloon dilation", "endoscopic papillary large balloon dilation" and "endoscopic sphincterotomy". The titles and abstracts of literature research was independently conducted and screened by two authors (Y.N.W., M.Q.). Discrepancies were resolved by consensus and a final decision was made by the third author (J.B.H.). 


\section{Study selection}

Inclusion criteria were: (1) both randomized controlled trials (RCTs) and retrospective cohort trials (RCSs) comparing ballooning dilation duration with $\leq 30$ seconds vs ballooning dilation duration with $\geq 1$ minute; (2) trials enrolling CBD stones removed by ERCP for patients with choledocholithiasis; (3) trials following Cotton consensus guideline to assess post-ERCP complications[17]; (4) full-length article. And exclusion criteria were: (1) systematic reviews, metaanalysis and case reports involved in commenting EPBD duration; (2) trials comparing both groups with long duration ( $\geq 1$ minute) of balloon dilation; (3) trials aiming for other diseases, such as biliary stenosis or biliary tumor.

\section{Data extraction and quality assessment}

Extracted outcome includes from included studies: (1) total CBD stone clearance rate during ERCP; (2) CBD stones clearance rate during the first ERCP; (3) frequency of mechanical lithotripsy used; (4) frequency of overall post-procedural complication; (5) frequency of PEP, bleeding, perforation and cholangitis.

According to Cochrane guideline and consensus, we collected baseline clinical data from included research and converted them to a common data extraction form[16, 18]. Baseline information includes first author, publication year, trial design, country, male proportion, patients age, past history of cholecystectomy, existence of periampullary diverticula, balloon diameter, proportion of single CBD stone, size of the largest CBD stone, CBD diameter, the usage of EST or postERCP pancreatic stents. Data were independently extracted by two reviewers (Y.N.W., M.Q.) and confirmed by a third reviewer (J.B.H.) for agreement. We tried to contact the authors if detailed supplementary information of included trials cannot be accurately extracted.

The quality of RCTs was evaluated on the basis of Cochrane risk-of-bias criteria (Version 5.1.0)[16]. The following 7 points were assessed: (1) randomization sequence generation; (2) allocation concealment; (3) blinding of participants and researchers; (4) blinding of the outcome assessors; (5) selective outcome reporting; (6) attrition bias; (7) other biases. The quality of RCSs was assessed by using the nine-point Newcastle-Ottawa Scale (NOS) scale to assess the following three fundamental aspects of methodology: study participant selection (0-4), confounder adjustment (0-2), and outcome indicator determination $(0-3)$. A RCS with $\geq 7$ points NOS score was defined as a highly qualified study and included in final analysis[19].

\section{Statistical analysis}

A pooled odds ratio (OR) and $95 \%$ confidence interval $(\mathrm{Cl})$ was adopted in our statistical analysis. The pooled continuous data are presented as a form of means \pm standard error. Two-tailed with $\mathrm{P}<0.05$ was considered statistically significant. Subgroup analysis was performed to assess the effect of prior EST, large balloon and 5-minute strategy on the efficacy and safety of EPBD. We also assessed publication bias via funnel plot asymmetry and Egger test[20]. $\mathrm{P} \geq$ 0.1 was considered to indicate no significant publication bias. The statistical heterogeneity was evaluated by Higgins $\mathrm{I}^{2}$ statistic and the chi square-based $\mathrm{Q}$ statistic [21]. Heterogeneity was defined to be not significant when $P>0.1$ and $I^{2}<50 \%$. Fixed-effect model was selected without significant heterogeneity and random-effect model was used if heterogeneity is significant. Sensitivity analysis was also performed to confirm the stability of our results. All the analyses described above were performed with STATA 15.0 statistical software (Stata Corp., College Station, TX, USA).

\section{Results}

\section{Literature search and basic characteristics of included trials}

A total of 644 potentially eligible articles were identified from PubMed, EMBASE and Cochrane Library database. After screening titles, abstracts and full text, seven full-length published trials were ultimately selected (Fig. 1), including five RCTs (2263 subjects)[22-26] and two nonrandomized studies (1255 subjects) $[15,27]$. A summary of detailed baseline characteristics from all included trials are reviewed and presented in Table 1. 
Table 1

Baseline characteristics of included studies. RCT, randomized controlled trial; RCS, retrospective cohort study; EPBD, endoscopic papillary balloon dilation; EP endoscopic papillary large balloon dilation; ESBD, endoscopic sphincterotomy plus balloon dilation; ESLBD, endoscopic sphincterotomy plus large balloon dili

NA, not available.

\begin{tabular}{|c|c|c|c|c|c|c|c|c|c|c|c|c|c|c|c|}
\hline \multirow[t]{2}{*}{$\begin{array}{l}\text { Author } \\
\text { with } \\
\text { country }\end{array}$} & \multirow[t]{2}{*}{$\begin{array}{l}\text { Study } \\
\text { type }\end{array}$} & \multirow[t]{2}{*}{$\begin{array}{l}\text { Centers } \\
\text { involved, } \\
\text { n }\end{array}$} & \multirow{2}{*}{$\begin{array}{l}\text { Study } \\
\text { design } \\
\text { (sample } \\
\text { size, n) }\end{array}$} & \multicolumn{2}{|c|}{ Male, n (\%) } & \multicolumn{2}{|l|}{ Age, y } & \multicolumn{2}{|c|}{$\begin{array}{l}\text { Balloon } \\
\text { diameter, mm }\end{array}$} & \multicolumn{2}{|c|}{$\begin{array}{l}\text { Maximum } \\
\text { size of } \\
\text { stones, mm }\end{array}$} & \multicolumn{2}{|c|}{$\begin{array}{l}\text { CBD diameter, } \\
\mathrm{mm}\end{array}$} & \multicolumn{2}{|c|}{$\begin{array}{l}\text { Multiple ston€ } \\
(\%)\end{array}$} \\
\hline & & & & $<30 s$ & $>1 \mathrm{~min}$ & $<30 \mathrm{~s}$ & $\begin{array}{l}> \\
1 \mathrm{~min}\end{array}$ & $<30 s$ & $\begin{array}{l}> \\
1 \mathrm{~min}\end{array}$ & $\begin{array}{l}< \\
30 \mathrm{~s}\end{array}$ & $\begin{array}{l}> \\
1 \mathrm{~min}\end{array}$ & $<30 \mathrm{~s}$ & $\begin{array}{l}> \\
1 \mathrm{~min}\end{array}$ & $<30 \mathrm{~s}$ & $>1$ \\
\hline $\begin{array}{l}\text { Tsujino } \\
2008 \\
\text { (Japan) }\end{array}$ & RCS & 6 & $\begin{array}{l}\text { EPBD } \\
15 \mathrm{~s} \\
(324) \\
\text { vs } \\
2 \text { min } \\
(324)\end{array}$ & $\begin{array}{l}191 \\
(59.0 \%)\end{array}$ & $\begin{array}{l}191 \\
(59.0 \%)\end{array}$ & $\begin{array}{l}70.0 \\
\pm \\
11.83\end{array}$ & $\begin{array}{l}69.3 \\
\pm \\
12.83\end{array}$ & NA & NA & $\begin{array}{l}7.1 \\
\pm 3.8\end{array}$ & $\begin{array}{l}7.2 \pm \\
5.0\end{array}$ & $\begin{array}{l}10.6 \\
\pm 5.2\end{array}$ & $\begin{array}{l}11.1 \\
\pm 5.5\end{array}$ & NA & NA \\
\hline $\begin{array}{l}\text { Bang } \\
2010 \\
\text { (Korea) }\end{array}$ & RCT & 1 & $\begin{array}{l}\text { EPBD } \\
20 \mathrm{~s} \\
(35) \mathrm{vs} \\
1 \mathrm{~min} \\
(35)\end{array}$ & $\begin{array}{l}16 \\
(45.7 \%)\end{array}$ & $\begin{array}{l}19 \\
(54.3 \%)\end{array}$ & $\begin{array}{l}63.3 \\
\pm 13.6\end{array}$ & $\begin{array}{l}66.2 \\
\pm 17.4\end{array}$ & $\begin{array}{l}9.6 \pm \\
2.4\end{array}$ & $\begin{array}{l}9.7 \pm \\
2.6\end{array}$ & $\begin{array}{l}8.2 \\
\pm 3.3\end{array}$ & $\begin{array}{l}8.1 \pm \\
3.5\end{array}$ & $\begin{array}{l}13.1 \\
\pm 4.2\end{array}$ & $\begin{array}{l}12.3 \\
\pm 3.1\end{array}$ & $\begin{array}{l}14 \\
(40 \%)\end{array}$ & $\begin{array}{l}19 \\
(54\end{array}$ \\
\hline $\begin{array}{l}\text { Paspatis } \\
2013 \\
\text { (Greece) }\end{array}$ & RCT & 1 & $\begin{array}{l}\text { ESLBD } \\
30 \mathrm{~s} \\
(64) \mathrm{vs} \\
1 \mathrm{~min} \\
(60)\end{array}$ & $\begin{array}{l}23 \\
(35.9 \%)\end{array}$ & $\begin{array}{l}27 \\
(45.0 \%)\end{array}$ & $\begin{array}{l}75.1 \\
\pm 11.9\end{array}$ & $\begin{array}{l}74.7 \\
\pm 13.2\end{array}$ & $\begin{array}{l}15.08 \\
\pm 1.12\end{array}$ & $\begin{array}{l}15.22 \\
\pm 1.45\end{array}$ & $\begin{array}{l}15.8 \\
\pm 4.3\end{array}$ & $\begin{array}{l}15.7 \\
\pm 5.2\end{array}$ & $\begin{array}{l}17.1 \\
\pm 4.8\end{array}$ & $\begin{array}{l}18.4 \\
\pm 5.4\end{array}$ & NA & NA \\
\hline $\begin{array}{l}\text { Sun } \\
2014 \\
\text { (China) }\end{array}$ & RCT & 1 & $\begin{array}{l}\text { EPLBD } \\
0 \mathrm{~s}(27) \\
\mathrm{vs} \\
1 \mathrm{~min}+ \\
5 \mathrm{~min} \\
(62)\end{array}$ & $\begin{array}{l}12 \\
(44.4 \%)\end{array}$ & $\begin{array}{l}32 \\
(51.6 \%)\end{array}$ & $\begin{array}{l}63.3 \\
\pm \\
10.71\end{array}$ & $\begin{array}{l}69.2 \\
\pm 11.1\end{array}$ & $\begin{array}{l}15.33 \\
\pm 1.78\end{array}$ & $\begin{array}{l}14.7 \\
\pm 1.87\end{array}$ & $\begin{array}{l}18.7 \\
\pm 3.6\end{array}$ & $\begin{array}{l}16.2 \\
\pm 3.6\end{array}$ & $\begin{array}{l}17.6 \\
\pm 3.2\end{array}$ & $\begin{array}{l}16.9 \\
\pm 3.6\end{array}$ & $\begin{array}{l}24 \\
(88.9 \%)\end{array}$ & $\begin{array}{l}53 \\
(85\end{array}$ \\
\hline $\begin{array}{l}\text { Shavakhi } \\
2015 \\
\text { (Iran) }\end{array}$ & RCT & 1 & $\begin{array}{l}\text { ESBD } \\
5 \mathrm{~s}(31) \\
\text { vs } \\
1 \mathrm{~min} \\
(29)\end{array}$ & $\begin{array}{l}10 \\
(32.3 \%)\end{array}$ & $\begin{array}{l}14 \\
(48.3 \%)\end{array}$ & $\begin{array}{l}55.74 \\
\pm 20.7\end{array}$ & $\begin{array}{l}54.97 \\
\pm \\
19.83\end{array}$ & $\begin{array}{l}12.22 \\
\pm 2.16\end{array}$ & $\begin{array}{l}12.22 \\
\pm 2.08\end{array}$ & $\begin{array}{l}7.74 \\
\pm \\
2.96\end{array}$ & $\begin{array}{l}8.1 \pm \\
3.65\end{array}$ & $\begin{array}{l}12.97 \\
\pm 4.54\end{array}$ & $\begin{array}{l}12.86 \\
\pm 4.8\end{array}$ & $\begin{array}{l}3 \\
(9.7 \%)\end{array}$ & $\begin{array}{l}7 \\
(24\end{array}$ \\
\hline $\begin{array}{l}\text { Hakuta } \\
2017 \\
\text { (Japan) }\end{array}$ & RCS & 5 & $\begin{array}{l}\text { EPBD } \\
15 \mathrm{~s} \\
(450) \\
\text { Vs } \\
5 \mathrm{~min} \\
(157)\end{array}$ & $\begin{array}{l}272 \\
(60.4 \%)\end{array}$ & $\begin{array}{l}106 \\
(67.5 \%)\end{array}$ & $\begin{array}{l}73.5 \\
\pm 15.0\end{array}$ & $\begin{array}{l}75.12 \\
\pm 17\end{array}$ & NA & NA & $\begin{array}{l}6 \pm \\
5.67\end{array}$ & $\begin{array}{l}5 \pm \\
3.17\end{array}$ & $\begin{array}{l}10 \pm \\
4.17\end{array}$ & $\begin{array}{l}9 \pm \\
2.67\end{array}$ & NA & NA \\
\hline $\begin{array}{l}\text { Meng } \\
2019 \\
\text { (China) }\end{array}$ & RCT & 15 & $\begin{array}{l}\text { ESBD } \\
0 s+ \\
30 \mathrm{~s} \\
(755) \\
\text { vs } \\
1 \mathrm{~min}+ \\
3 \mathrm{~min}+ \\
5 \mathrm{~min} \\
(1165)\end{array}$ & $\begin{array}{l}343 \\
(45.4 \%)\end{array}$ & $\begin{array}{l}572 \\
(49.1 \%)\end{array}$ & $\begin{array}{l}64.5 \\
\pm 17.1\end{array}$ & $\begin{array}{l}64.7 \\
\pm 16.1\end{array}$ & NA & NA & $\begin{array}{l}10 \pm \\
3.0\end{array}$ & $\begin{array}{l}10 \pm \\
3.3\end{array}$ & $\begin{array}{l}12 \pm \\
3.7\end{array}$ & $\begin{array}{l}12 \pm \\
3.7\end{array}$ & $\begin{array}{l}387 \\
(51.3 \%)\end{array}$ & $\begin{array}{l}62 \\
(53\end{array}$ \\
\hline
\end{tabular}

Five RCTs were conducted in following countries: two in China; one in Korea, Greece and Iran, respectively. Both two RCSs are from Japan. Three trials were multicentered research including two $\operatorname{RCSs}[15,26,27]$. There are three trials involved in endoscopic sphincterotomy with balloon dilation $(E S B D)[23,25,26]$ including one trial implementing endoscopic sphincterotomy with large balloon dilation (ESLBD)[23]. Large balloon dilation was used in two trials[23, 24]. Five-minute strategy in EPBD dilation was involved in three trials[24, 26, 27]. No significant publication bias was identified by funnel plot (Figure S1) and Egger's test $(P=0.45$, Figure $S 2)$.

\section{Efficacy comparison}

All included trials reported total CBD stone clearance rate across all ERCP sessions, which was compared between short duration group and long duration group. As shown in Fig. 2, pooled $\mathrm{OR}(0.98,95 \% \mathrm{Cl}[0.73,1.31], \mathrm{P}=0.89)$ demonstrated no significance in the rate of total stone clearance without significant heterogeneity $\left(I^{2}=0 \%, P=0.73\right)$. And the rate of complete stone removal during the first ERCP procedure was assessed between two groups after excluding three RCTs without reporting relevant data. No significant heterogeneity was shown $\left(I^{2}=32.4 \%, P=0.22\right)$. Figure 3 revealed that $\geq 1$-minute duration group significantly had higher successful rate of complete stone clearance than $<30$-second duration group during the first ERCP session (OR $0.61,95 \% \mathrm{Cl}[0.48$, 0.77], $P<0.001)$. A total of five studies[15, 22, 24, 26, 27] were included to compare the frequency of mechanical lithotripsy use during ERCP. The heterogeneity was statistically significant $\left(I^{2}=62.9 \%, P=0.03\right)$. In spite of no significance, $\leq 30$-second duration group implemented mechanical lithotripsy more frequently compared with $\geq 1$-minute duration group $(\mathrm{OR} 1.22,95 \% \mathrm{Cl}[0.79,1.89], \mathrm{P}=0.37)$ by using a random-effect model (Fig. 4). The information of procedure time 
was available in only three studies[24, 26, 27]. Obvious heterogeneity was exhibited $\left(I^{2}=91.3 \%, P<0.001\right)$. The weighted mean difference $(W M D)$ was -1.61 $(95 \% \mathrm{Cl}[-7.05,3.83], \mathrm{P}=0.56)$ measured by the random-effect model.

\section{Safety comparison}

The rate of overall early complications was compared between two groups. Our meta-analysis without significant heterogeneity $\left(I^{2}=0 \%, P=0.61\right)$ revealed short duration group had a relatively lower rate of early complication although statistical significance was not generated $(0 \mathrm{R} 0.85,95 \% \mathrm{Cl}[0.70,1.04], \mathrm{P}=0.11$, Fig. 5). In addition, common complications were analyzed, respectively. No significance between two groups was demonstrated in the rate of PEP (OR 0.81 , $95 \% \mathrm{Cl}[0.63,1.03], \mathrm{P}=0.09$, Fig. 6), hemorrhage (OR 0.86, 95\% Cl [0.30, 2.44], $\mathrm{P}=0.77)$, perforation (OR $0.54,95 \% \mathrm{Cl}[0.14,2.14], \mathrm{P}=0.38)$ and cholangitis $(\mathrm{OR}$ $1.15,95 \% \mathrm{Cl}[0.80,1.66], \mathrm{P}=0.44)$. And all analysis of common complications showed no significant heterogeneity.

\section{Subgroup analysis}

As shown in Table 2, Subgroup analyses were conducted to investigate the effect of EST, use of large balloon dilation and 5-minute strategy in the efficacy and safety between two groups. The results demonstrated that there was no significant difference $(\mathrm{OR} 0.96,95 \% \mathrm{Cl}[0.55,1.69], \mathrm{P}=0.90)$ between $\leq 30$-second duration and $\geq 1$-minute duration in the subgroup taking balloon dilation with prior EST, which indicated prior EST potentially increased the efficacy of stone removal in short duration group during the first ERCP session. And the subgroup analysis of EPLBD, short duration group had longer procedure time than long duration group (WMD $13.10,95 \% \mathrm{Cl}[9.39,16.81], \mathrm{P}<0.001)$ but this result was produced by only one trials which was also the potential source of heterogeneity. Additionally, no significant difference was shown in the rate of overall early complications $(\mathrm{OR} 0.94,95 \% \mathrm{Cl}[0.75,1.18], \mathrm{P}=0.58)$ and pancreatitis $(\mathrm{OR} 0.90,95 \% \mathrm{Cl}[0.68,1.18], \mathrm{P}=0.44)$ between $<30$-second duration group and $\geq 1$-minute duration group with 5 -minute duration involvement. This result implied that 5-minute duration could reduce the risk of early complications and PEP. Besides, prior EST, large balloon dilation and 5-minute duration had no significant effect on other outcomes concerning the efficacy and safety of EPBD.

Table 2

Subgroup analysis for the effect of prior EST, large balloon dilation and 5-minute strategy on the efficacy and safety of EPBD between short dilation duration group and long dilation duration group. EST, endoscopic sphincterotomy; ERCP, Endoscopic Retrograde Cholangiopancreatography.

\begin{tabular}{|c|c|c|c|c|c|c|c|}
\hline \multirow[t]{3}{*}{ Results } & \multirow[t]{3}{*}{ Outcome } & \multicolumn{6}{|c|}{ Subgroup analysis (OR/WMD [95\%Cl]) } \\
\hline & & \multicolumn{2}{|l|}{ Prior EST } & \multicolumn{2}{|c|}{ Large balloon dilation } & \multicolumn{2}{|c|}{$\begin{array}{l}\text { 5-minute strategy } \\
\text { involvement }\end{array}$} \\
\hline & & Yes & No & Yes & No & Yes & No \\
\hline \multirow[t]{4}{*}{ Efficacy } & Total stone clearance rate & $\begin{array}{l}1.04[0.76 \\
1.43] \\
P=0.82\end{array}$ & $\begin{array}{l}0.73[0.35 \\
1.51] \\
P=0.393\end{array}$ & $\begin{array}{l}1.08[0.40 \\
2.93] \\
P=0.88\end{array}$ & $\begin{array}{l}0.97[0.72 \\
1.31] \\
P=0.85\end{array}$ & $\begin{array}{l}0.98[0.70 \\
1.36] \\
P=0.89\end{array}$ & $\begin{array}{l}0.98[0.53, \\
1.82] \\
P=0.96\end{array}$ \\
\hline & $\begin{array}{l}\text { Rate of complete stone removal during first } \\
\text { ERCP session }\end{array}$ & $\begin{array}{l}0.96[0.55 \\
1.69] \\
P=0.90\end{array}$ & $\begin{array}{l}0.55[0.42 \\
0.72] \\
P<0.001\end{array}$ & \multicolumn{2}{|c|}{ No Subgroup analysis } & $\begin{array}{l}0.60[0.42 \\
0.86] \\
P=0.005\end{array}$ & $\begin{array}{l}0.61[0.44, \\
0.85] \\
P=0.003\end{array}$ \\
\hline & Mechanical lithotripsy use & $\begin{array}{l}1.00[0.76 \\
1.32] \\
P=0.99 *\end{array}$ & $\begin{array}{l}1.39[0.63 \\
3.04] \\
P=0.41\end{array}$ & $\begin{array}{l}1.17[0.32 \\
4.29] \\
P=0.81^{\star}\end{array}$ & $\begin{array}{l}1.24[0.76 \\
2.02] \\
P=0.39\end{array}$ & $\begin{array}{l}1.49[0.69 \\
3.22] \\
P=0.31\end{array}$ & $\begin{array}{l}0.88[0.62, \\
1.25] \\
P=0.47\end{array}$ \\
\hline & Procedure time & $\begin{array}{l}0.00[-1.42, \\
1.42] \\
P=1.00 *\end{array}$ & $\begin{array}{l}-2.48[-13.36, \\
8.40] \\
P=0.66\end{array}$ & $\begin{array}{l}3.10[-0.61 \\
6.81] \\
P=0.10 *\end{array}$ & $\begin{array}{l}-3.85[-11.68, \\
3.99] \\
P=0.34\end{array}$ & \multicolumn{2}{|c|}{ No subgroup analysis } \\
\hline \multirow[t]{5}{*}{ Safety } & Overall complications & $\begin{array}{l}0.90[0.71 \\
1.14] \\
P=0.364\end{array}$ & $\begin{array}{l}0.75[0.53 \\
1.08] \\
P=0.12\end{array}$ & $\begin{array}{l}0.74[0.29 \\
1.87] \\
P=0.53\end{array}$ & $\begin{array}{l}0.86[0.70 \\
1.05] \\
P=0.14\end{array}$ & $\begin{array}{l}0.94[0.75 \\
1.18] \\
P=0.58\end{array}$ & $\begin{array}{l}0.61[0.40, \\
0.93] \\
P=0.02\end{array}$ \\
\hline & Pancreatits & $\begin{array}{l}0.84[0.62 \\
1.13] \\
P=0.24\end{array}$ & $\begin{array}{l}0.74[0.47 \\
1.15] \\
P=0.18\end{array}$ & $\begin{array}{l}0.94[0.13 \\
6.86] \\
P=0.95^{\star}\end{array}$ & $\begin{array}{l}0.81[0.63 \\
1.03] \\
P=0.09\end{array}$ & $\begin{array}{l}0.90[0.68 \\
1.18] \\
P=0.44\end{array}$ & $\begin{array}{l}0.52[0.30, \\
0.92] \\
P=0.03\end{array}$ \\
\hline & Hemorrhage & $\begin{array}{l}0.67[0.19 \\
2.30] \\
P=0.52\end{array}$ & $\begin{array}{l}1.84[0.19 \\
17.66] \\
P=0.60\end{array}$ & $\begin{array}{l}0.45[0.08 \\
2.56] \\
P=0.37 *\end{array}$ & $\begin{array}{l}1.31[0.33 \\
5.15] \\
P=0.70\end{array}$ & $\begin{array}{l}1.03[0.22 \\
4.94] \\
P=0.97\end{array}$ & $\begin{array}{l}0.74[0.18, \\
3.03] \\
P=0.67\end{array}$ \\
\hline & Perforation & $\begin{array}{l}0.71[0.09 \\
5.61] \\
P=0.75\end{array}$ & $\begin{array}{l}0.43[0.07 \\
2.73] \\
P=0.37\end{array}$ & $\begin{array}{l}0.94[0.06 \\
15.32] \\
P=0.96 *\end{array}$ & $\begin{array}{l}0.45[0.09 \\
2.25] \\
P=0.33\end{array}$ & $\begin{array}{l}0.42[0.05 \\
3.59] \\
P=0.43\end{array}$ & $\begin{array}{l}0.65[0.11, \\
3.91] \\
P=0.64\end{array}$ \\
\hline & Cholangitis & $\begin{array}{l}1.20[0.79 \\
1.82] \\
P=0.39\end{array}$ & $\begin{array}{l}1.02[0.50 \\
2.11] \\
P=0.95\end{array}$ & $\begin{array}{l}1.43[0.23 \\
8.85] \\
P=0.70 *\end{array}$ & $\begin{array}{l}1.14[0.79 \\
1.65] \\
P=0.48\end{array}$ & $\begin{array}{l}1.20[0.80 \\
1.78] \\
P=0.38\end{array}$ & $\begin{array}{l}0.99[0.42, \\
2.30] \\
P=0.97\end{array}$ \\
\hline
\end{tabular}


EPBD has been widely accepted as a potential method for preserving the function of SO during ERCP but no consensus is established to define explicit duration of EPBD. Our review and meta-analysis were the first systematic investigation to figure out the effect of EPBD duration on the efficacy and safety of therapeutic ERCP for choledocholithiasis by comparing short duration dilation ( $\leq 30$ seconds) and long duration ( $\geq 1$ minute) dilation. There were also some potentially confounding factors including preceding EST, the use of large balloon dilation and 5-minute long duration. Subgroup analyses were taken to identify their effect on the efficacy and safety of ERCP for CBD stones. Both RCTs and RCSs were included in our analysis due to limited number of trials showing the comparison between short and long dilation time.

During the first ERCP session, a significantly higher efficiency in complete removal of CBD stones was shown in the EPBD group with long dilation duration. Intriguingly, subgroup analysis indicated that the efficacy of stone removal during the first ERCP session was improved by preceding EST especially for $\leq 30$ second dilation because there was no significance in the rate of stone removal between short- and long-time dilation with preceding EST. Recently, a network meta-analysis has identified ESBD has a significant higher rate of stone removal during the first endoscopic session compared with EST and EPBD[28]. Besides, $\geq 1$-minute dilation duration of EPBD demonstrated a trend of higher rate of total stone clearance and less frequency of mechanical lithotripsy use although no significance was verified.

After comparing the efficiency between two groups, we compared a total rate of overall early complications. The result revealed that short duration of EPBD could have a trend to reduce the risk of overall complications and pancreatitis compared with long duration of EPBD. Our result was opposed to an earlier meta-analysis demonstrating duration of EPBD is inversely associated with pancreatitis risk[13]. Their results were mainly based on indirect comparison with EST and only one trial was included in the comparison between short and long EPBD despite Bayesian statistical approach was used. And subgroup analysis implied that five-minute duration in the group with long duration dilation could potentially reduce the incidence of overall complications and PEP. Our result agreed on the results of the randomized research illustrating that five-minute duration of EPBD triggered a significant lower risk of overall complications and pancreatitis than one-minute duration of EPBD[29]. There are many disputes on the issue of the relationship between EPBD time and subsequent complications. Short duration dilation may restrict the enlargement and expansion of SO, which increase a risk of PEP[29]. Other influence factors include difficult biliary cannulation and operation without gentleness, which could induce papillary irritation[30]. But now prophylactic pancreatic stents have been proven to effectively reduce the incidence of PEP[31]. EPBD is an effective approach to avoid the short-term complications of perforation and bleeding. And hemorrhage, perforation and cholangitis did not show any significance between two groups in our results.

No significant heterogeneity was proven among most results. But significant heterogeneity existed in the comparison of mechanical lithotripsy use and procedure time. Selection bias in a RCS was figured out as the main source of heterogeneity[27]. All included patients undergoing EPBD with short duration were from 2008 to 2013 and all EPBD operation with long duration were performed after 2013. Lack of operational experience and imperfect technique and equipment may contribute to more frequent use of lithotripsy and longer procedure time.

The results of our meta-analysis could provide an instructive clinical significance but also have some inevitable limitations. Firstly, relatively insufficient number of trials and small sample size may potentially reduce the quality and reliability of the evidence. More RCTs are required to identify the better choice for EPBD duration. Secondly, one to three-minute or five-minute duration were mainstream strategies of long EPBD. The comparative results could also be impacted by the time span in group with long duration of EPBD although no significant heterogeneity was proven. In our analysis, subgroup analysis for fiveminute strategy was used to discuss the effect of different time choice in the group with long EPBD duration on final results and conclusions. Thirdly, the safety of EPBD duration we discussed only referred to short-term/early complications. Long-term/late complications also are required to be focused on. Only one RCT systematically reported no significant difference in recurrent choledocholithiasis and hepatobiliary complications between 1-minute and 5-minute EPBD group[32]. More comparative analyses of late complications after EPBD should be conducted in the future.

\section{Conclusions}

Long duration ( $\geq 1$ minute) could significantly increase the efficacy of EPBD in stone removal rate during first ERCP session compared with short duration. Long duration EPBD group demonstrated higher rate of total CBD stone clearance and less mechanical lithotripsy although not significantly. Moreover, short EPBD contributed to less overall complications and PEP than long EPBD but not significantly. Prophylactic pancreatic stent placement could be an effective strategy against PEP nowadays. For long duration of EPBD, 5-minute duration may potentially reduce the rate of overall complications and pancreatitis.

\section{Abbreviations}

EPBD, Endoscopic papillary balloon dilation; EST, endoscopic sphincterotomy; ERCP, endoscopic retrograde cholangiopancreatography; CBDS, common bile duct stones; SO, sphincter of Oddi; PEP, post-ERCP pancreatitis; ESGE, European Society of Gastrointestinal Endoscopy; EPLBD, endoscopic papillary large balloon dilation; RCT, randomized controlled trial; RCS, retrospective cohort trial; ESBD, endoscopic sphincterotomy with balloon dilation; ESLBD, endoscopic sphincterotomy with large balloon dilation;

\section{Declarations}

Ethics approval and consent to participate Not applicable.

Consent for publication All authors read and approved the final manuscript.

Availability of data and material Not applicable.

Competing Interests The authors declare no conflict of interest. 
Funding This work was supported by Natural Science Foundation of China (No. 81760106) and Natural Science Foundation of Jiangxi Province (No. 20171BAB205012)

Authors' contributions HJB and WYN participated the design of this meta-analysis. WYN conducted literature searching, data extraction, data analysis and interpretation, drafted the manuscript. QM and HYZ participated in study design and the revision process of the manuscript. HJB participated in the critical revision of the manuscript.

Acknowledgement Not applicable.

\section{References}

1. Williams E, Beckingham I, El Sayed G, Gurusamy K, Sturgess R, Webster G, Young T: Updated guideline on the management of common bile duct stones (CBDS). Gut 2017, 66(5):765-782.

2. Zimmon DS, Falkenstein DB, Kessler RE: Endoscopic papillotomy for choledocholithiasis. N Engl J Med 1975, 293(23):1181-1182.

3. Staritz M, Ewe K, Meyer zum Buschenfelde KH: Endoscopic papillary dilation (EPD) for the treatment of common bile duct stones and papillary stenosis. Endoscopy 1983, 15 Suppl 1:197-198.

4. Testoni PA, Mariani A, Aabakken L, Arvanitakis M, Bories E, Costamagna G, Deviere J, Dinis-Ribeiro M, Dumonceau JM, Giovannini M et al: Papillary cannulation and sphincterotomy techniques at ERCP: European Society of Gastrointestinal Endoscopy (ESGE) Clinical Guideline. Endoscopy 2016, 48(7):657-683.

5. Mergener K, Baillie J: Endoscopic papillary balloon dilation for the management of common bile duct stones: experience of 226 cases. Gastrointest Endosc 1998, 48(4):444-446.

6. Yasuda I, Tomita E, Enya M, Kato T, Moriwaki H: Can endoscopic papillary balloon dilation really preserve sphincter of Oddi function? Gut 2001, 49(5):686691.

7. Weinberg BM, Shindy W, Lo S: Endoscopic balloon sphincter dilation (sphincteroplasty) versus sphincterotomy for common bile duct stones. Cochrane Database Syst Rev 2006(4):CD004890.

8. Maydeo A, Bhandari S: Balloon sphincteroplasty for removing difficult bile duct stones. Endoscopy 2007, 39(11):958-961.

9. Heo JH, Kang DH, Jung HJ, Kwon DS, An JK, Kim BS, Suh KD, Lee SY, Lee JH, Kim GH et al: Endoscopic sphincterotomy plus large-balloon dilation versus endoscopic sphincterotomy for removal of bile-duct stones. Gastrointest Endosc 2007, 66(4):720-726; quiz 768, 771.

10. Itoi T, Itokawa F, Sofuni A, Kurihara T, Tsuchiya T, Ishii K, Tsuji S, Ikeuchi N, Moriyasu F: Endoscopic sphincterotomy combined with large balloon dilation can reduce the procedure time and fluoroscopy time for removal of large bile duct stones. Am J Gastroenterol 2009, 104(3):560-565.

11. Ersoz G, Tekesin O, Ozutemiz AO, Gunsar F: Biliary sphincterotomy plus dilation with a large balloon for bile duct stones that are difficult to extract. Gastrointest Endosc 2003, 57(2):156-159.

12. G M, G P, L A, A A, M A, P A-S, M B, D D, JM D, JF G et al: Endoscopic management of common bile duct stones: European Society of Gastrointestinal Endoscopy (ESGE) guideline. 2019, 51(5):472-491.

13. Liao WC, Tu YK, Wu MS, Wang HP, Lin JT, Leung JW, Chien KL: Balloon dilation with adequate duration is safer than sphincterotomy for extracting bile duct stones: a systematic review and meta-analyses. Clin Gastroenterol Hepatol 2012, 10(10):1101-1109.

14. Feng Y, Zhu H, Chen X, Xu S, Cheng W, Ni J, Shi R: Comparison of endoscopic papillary large balloon dilation and endoscopic sphincterotomy for retrieval of choledocholithiasis: a meta-analysis of randomized controlled trials. J Gastroenterol 2012, 47(6):655-663.

15. Tsujino T, Kawabe T, Isayama H, Sasaki T, Kogure H, Togawa O, Arizumi T, Ito Y, Matsubara S, Yamamoto N et al: Efficacy and safety of low-pressured and short-time dilation in endoscopic papillary balloon dilation for bile duct stone removal. J Gastroenterol Hepato/ 2008, 23(6):867-871.

16. Cochrane Handbook for Systematic Reviews of Interventions, version 5.1 .0 (updated March 2011) [http://training.cochrane.org/handbook]

17. Cotton PB, Lehman G, Vennes J, Geenen JE, Russell RC, Meyers WC, Liguory C, NickI N: Endoscopic sphincterotomy complications and their management: an attempt at consensus. Gastrointest Endosc 1991, 37(3):383-393.

18. Hozo SP, Djulbegovic B, Hozo I: Estimating the mean and variance from the median, range, and the size of a sample. $B M C M e d$ Res Methodo/ $2005,5: 13$.

19. Wells GA SB, O'Connell D, et al: The Newcastle-Ottawa Scale (NOS) for assessing the quality of non-randomized studies in meta-analysis. Appl Eng Agric 2012, 18(6):727-734.

20. Egger M, Davey Smith G, Schneider M, Minder C: Bias in meta-analysis detected by a simple, graphical test. BMJ 1997, 315(7109):629-634.

21. Deeks JJ AD, Bradburn MJ: Statistical methods for examining heterogeneity and combining results from several studies in meta-analysis. Hoboken: Wiley-Blackwell 2008:285-312.

22. BW B, S J, DH L, JI L, JW L, KS K, HG K, YW S, YS K: The ballooning time in endoscopic papillary balloon dilation for the treatment of bile duct stones. The Korean journal of internal medicine 2010, 25(3):239-245.

23. GA P, K K, G T, E V, A T, A T, I C, M M, G C, E V et al: Sixty-versus thirty-seconds papillary balloon dilation after sphincterotomy for the treatment of large bile duct stones: a randomized controlled trial. Digestive Endoscopy 2013, 45(4):301-304.

24. Juan-Juan Sun HJ, Tao Mao, Xue-Guo Sun, Xin-Juan Kong, Qing-Xi Zhao, Zi-Bin Tian: Endoscopic papillary large balloon dilation after endoscopic sphincterotomy for treatment of common bile duct stones: Effects of different dilation durations. World chinese journal of digestology 2014, 22(11):15971601. 
25. A S, M M, MH A, A S, S S: A comparative study of one minute versus five seconds endoscopic biliary balloon dilation after small sphincterotomy in choleducolithiasis. Advanced biomedical research 2015, 4(undefined):28.

26. W M, JW L, K Z, W Z, Z W, L Z, H S, P X, W L, Q W et al: Optimal dilation time for combined small endoscopic sphincterotomy and balloon dilation for common bile duct stones: a multicentre, single-blinded, randomised controlled trial. The Lancet Gastroenterology \& Hepatology 2019, 4(6):425-434.

27. R H, T H, Y N, H I, H K, S M, T N, H Y, O T, S M et al: Multicenter retrospective and comparative study of 5-minute versus 15-second endoscopic papillary balloon dilation for removal of bile duct stones. Endoscopy International Open 2017, 5(11):E1027-E1034.

28. CH P, JH J, E N, EH K, MG K, JH K, endoscopy PSJG: Comparative efficacy of various endoscopic techniques for the treatment of common bile duct stones: a network meta-analysis. 2018, 87(1):43-57.e10.

29. WC L, CT L, CY C, JW L, JH C, MC T, JT L, MS W, endoscopy WHJG: Randomized trial of 1-minute versus 5-minute endoscopic balloon dilation for extraction of bile duct stones. 2010, 72(6):1154-1162.

30. SI J, GW Y, gastroenterology LDJWjo: Balloon dilation itself may not be a major determinant of post-endoscopic retrograde cholangiopancreatography pancreatitis. 2014, 20(45):16913-16924.

31. Choudhary A, Bechtold ML, Arif M, Szary NM, Puli SR, Othman MO, Pais WP, Antillon MR, Roy PK: Pancreatic stents for prophylaxis against post-ERCP pancreatitis: a meta-analysis and systematic review. Gastrointest Endosc 2011, 73(2):275-282.

32. YT K, HP W, CY C, JW L, JH C, MC T, gastroenterology LWJC, Association htocpjotAG: Comparable Long-term Outcomes of 1-Minute vs 5-Minute Endoscopic Papillary Balloon Dilation for Bile Duct Stones. 2017, 15(11):1768-1775

\section{Supplementary Figure Captions}

Figure S1. Funnel plot assessing for publication bias.

Figure S2. Egger's test assessing for publication bias.

\section{Figures}

\section{Initial searching screening 644 articles}

\section{Removal of 625 duplications and irrelevant articles}

\section{9 relevant articles selected and reviewed}

\section{7 studies included in the meta-analysis}


Study

ID

Tsujino et al. (2008)

Bang et al. (2010)

Paspatis et al. (2013)

Shavakhi et al. (2015)

Hakuta et al. (2017)

Meng et al. (2019)

Sun et al. (2014)

Overall (I-squared $=0.0 \%, p=0.725$ )

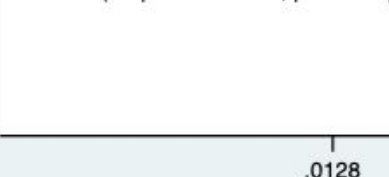

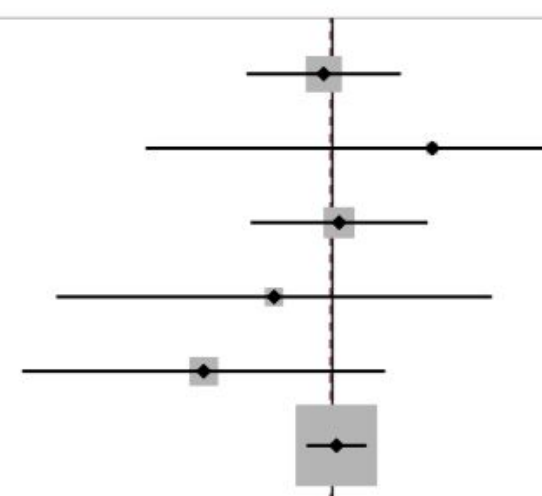

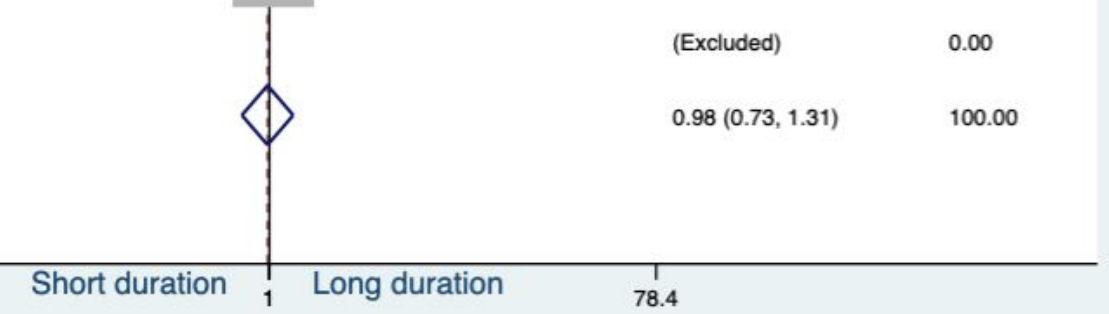

Figure 2

Forrest plot of included studies demonstrating the effect of EPBD duration on total CBD clearance rate during all ERCP sessions. 


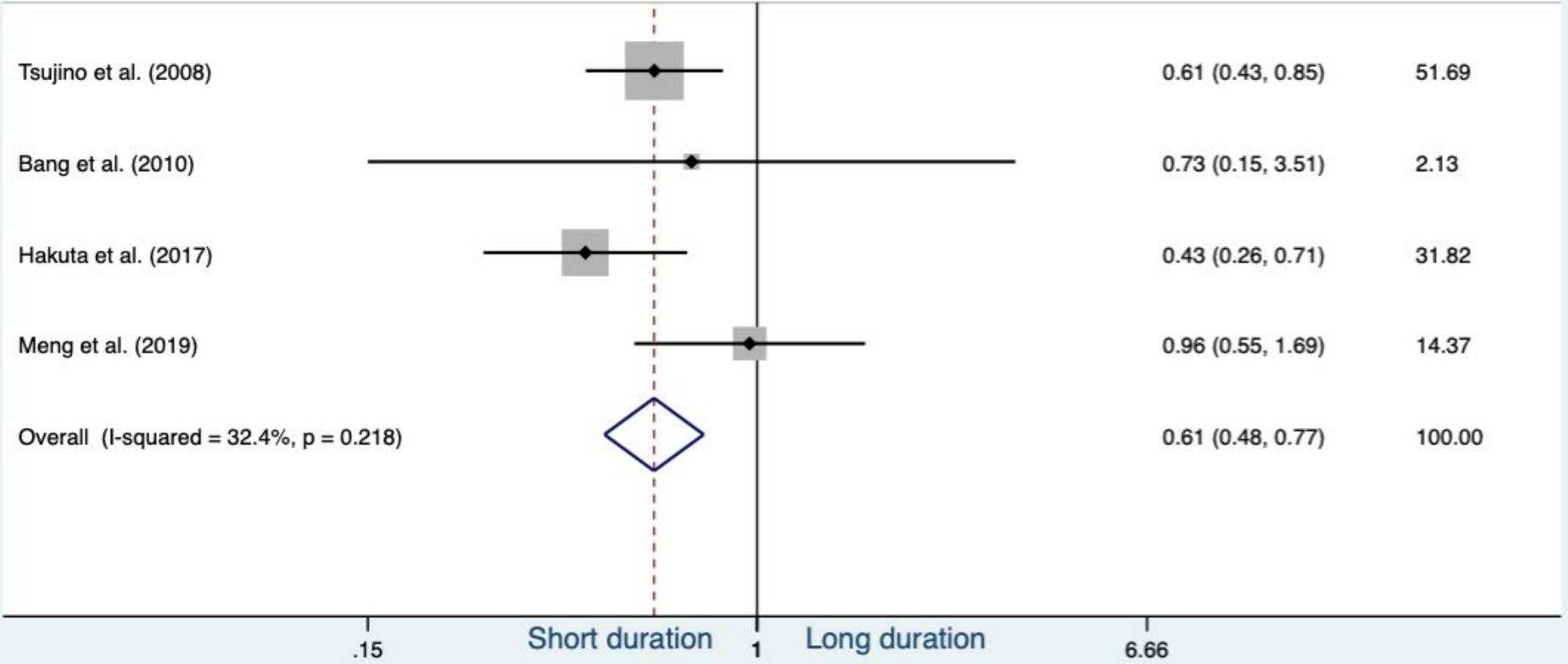

\section{Figure 3}

Forrest plot of included studies demonstrating the effect of EPBD duration on stone removal rate during the first ERCP session. 


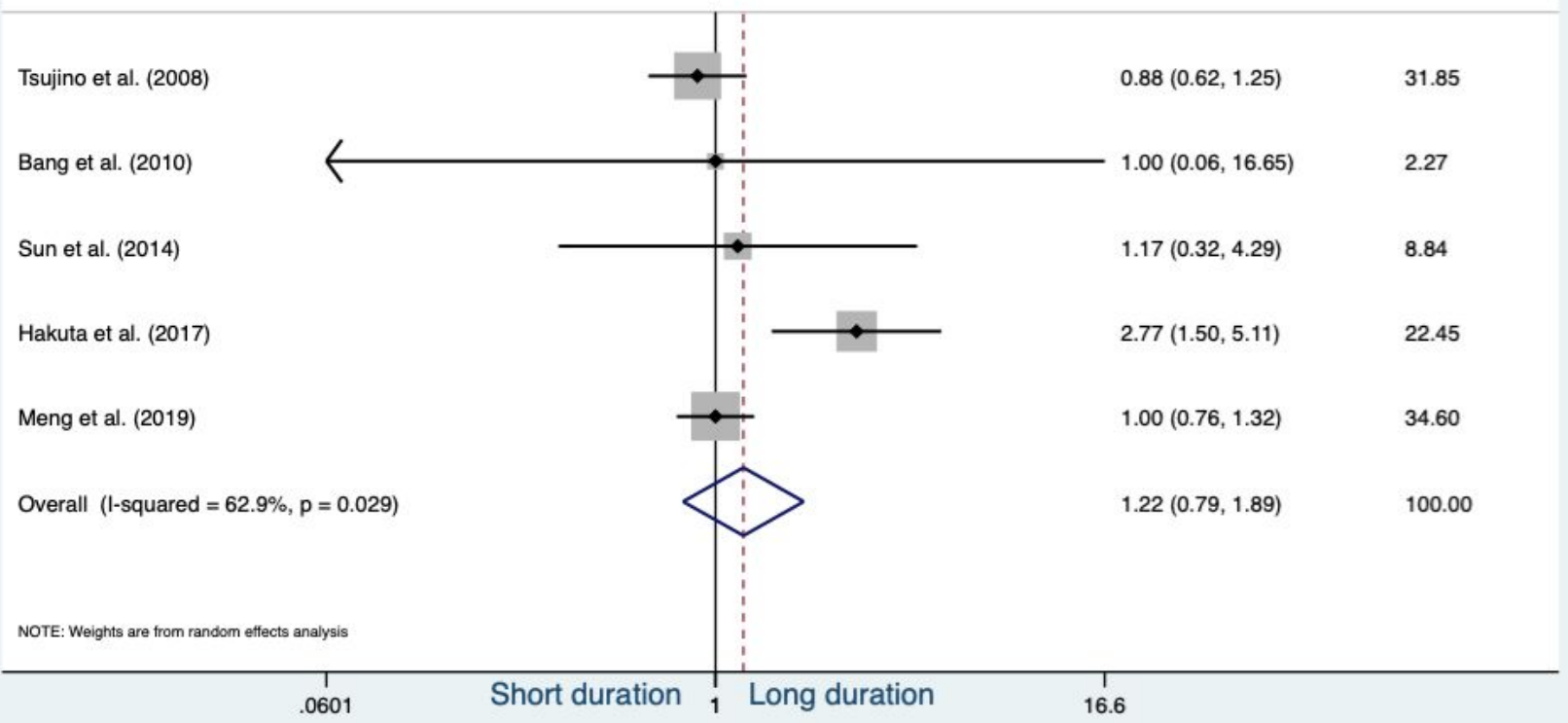

\section{Figure 4}

Forrest plot of included studies demonstrating the effect of EPBD duration on the frequency of mechanical lithotripsy use.

Study

ID

Tsujino et al. (2008)
Bang et al. (2010)
Paspatis et al. (2013)
Shavakhi et al. (2015)

Hakuta et al. (2017)

Meng et al. (2019)

Sun et al. (2014)

Overall (1-squared $=0.0 \%, p=0.608$ )
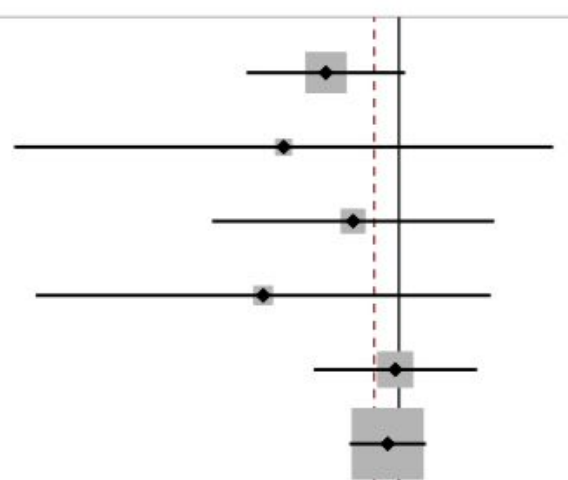

$0.93(0.72,1.19)$

60.40

(Excluded)

0.00

$0.85(0.70,1.04) \quad 100.00$

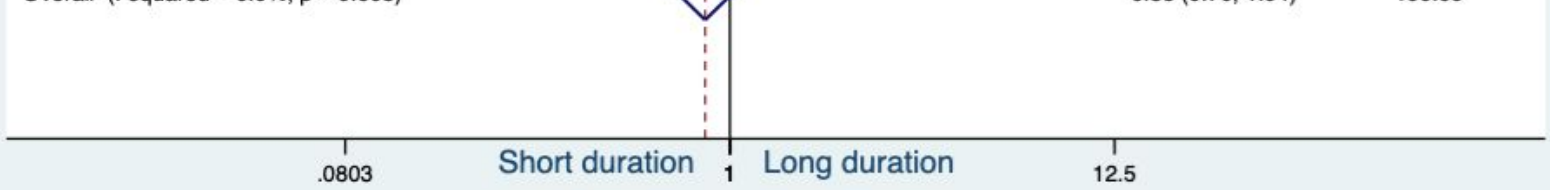


Study

ID

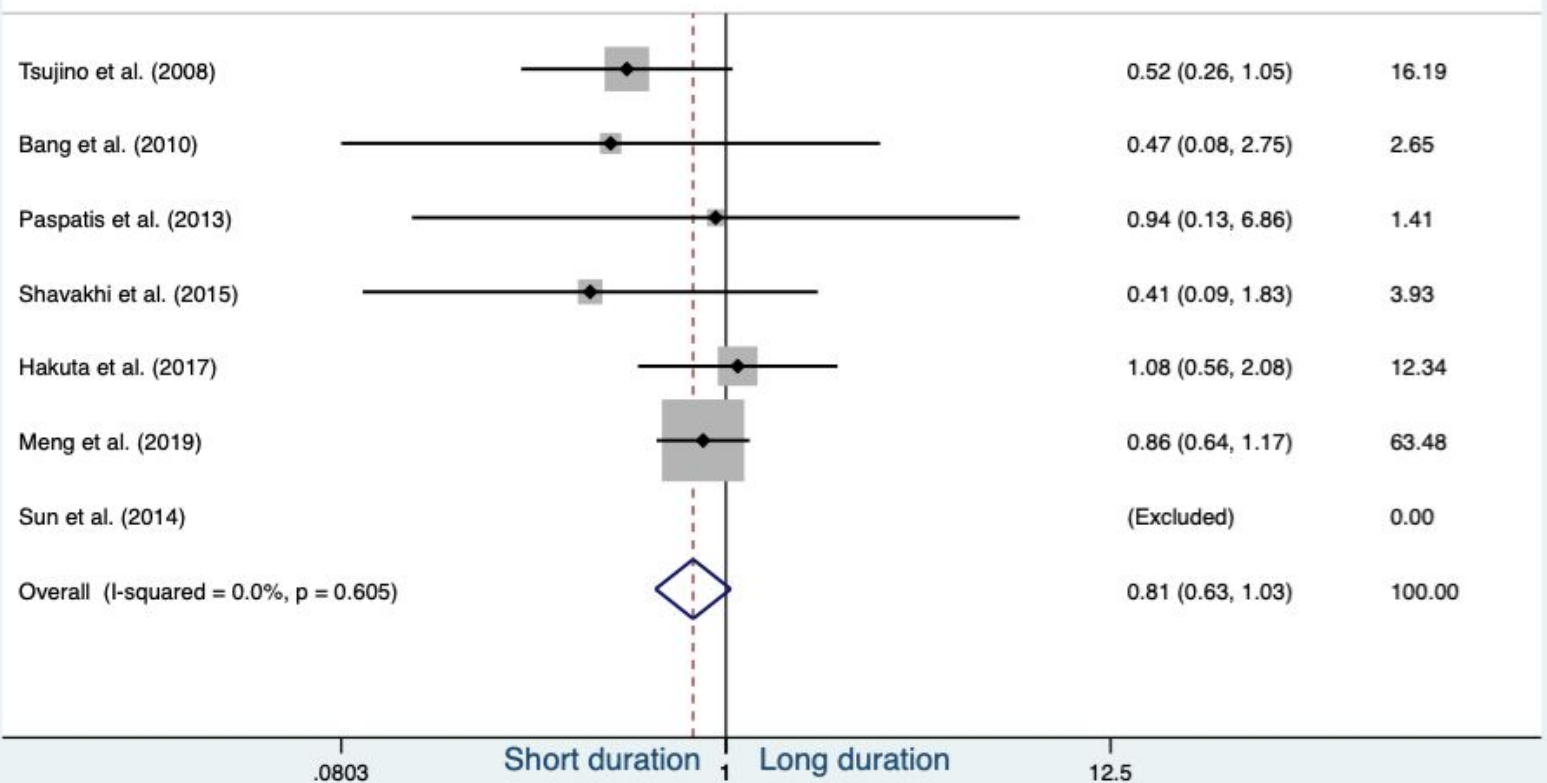

\section{Figure 6}

Forrest plot of included studies demonstrating the effect of EPBD duration on post-ERCP pancreatitis.

\section{Supplementary Files}

This is a list of supplementary files associated with this preprint. Click to download.

- 1PRISMANMAchecklist.docx

- Figures2.jpg

- FigureS1.jpg 